\title{
Acute Megakaryoblastic Leukaemia (AML M7): Rare association of Down syndrome and Haemoglobin E-Beta Thalassaemia.
}

\author{
Dr. Gautam Kumar Mandal ${ }^{1}$, Dr. Koushik Dewan ${ }^{1}$, Dr. Manik Mondal ${ }^{2}$, \\ Dr.Chinmoy Lath ${ }^{2}$, Dr. Rajarshi Basu ${ }^{2}$, Dr. Himel Bera ${ }^{3}$ \\ ${ }^{\prime}$ (Department of Pathology, Chittaranjan National Cancer Institute, India) \\ ${ }_{2}^{2}$ (Department of Pediatric Medicine, Nil Ratan Sircar Medical College and Hospital, The West Bengal \\ University of Health Sciences, India) \\ ${ }^{3}$ (Department of Pathology, Medical College, The West Bengal University of Health Sciences, India)
}

\begin{abstract}
Acute megakaryoblastic leukemia(AML-M7) is a rare type of pediatric AML. It represents approximately $1 \%$ of all leukemias during childhood \& has an incidence of $0.5 \%$ per million per year. In young children with Down syndrome, $A M L-M 7$ is the most common type of $A M L$. There is no association reported till date in between Down syndrome \& Haemoglobin E-Beta thalassaemia. In our case we got rare association of AML-M7 in a case of Down syndrome with Haemoglobin E-Beta thalassemia. This association may be incidental. But we are reporting this case for future review.
\end{abstract}

Keywords - Acute Megakaryoblastic Leukemia, AML-M7, Down syndrome, Haemoglobin E-Beta thalassaemia, rare association.

\section{INTRODUCTION}

Children with Down syndrome (constitutional trisomy 21) have a 10- to 20 -fold increased risk of developing acute leukemia[1,2,3]. Particularly frequent in this group, are 2 forms of myeloid leukemia. Acute megakaryoblastic leukemia (AMKL), a subtype of acute myeloid leukemia (FAB classification M7)[4] occurs with an incidence estimated to be 500-fold greater than in the general pediatric population[5]. Further, approximately $10 \%$ of newborn infants with Down syndrome develop transient leukemia (TL; reviewed in Zipursky [6] Gamis and Hilden[7]; and Taub and Ravindranath[8]), also referred to as transient myeloproliferative disorder and transient abnormal myelopoiesis, a form of megakaryoblastic leukemia specific for Down syndrome. In both, TL and AMKL, blasts accumulate in blood and bone marrow, and megakaryocytic differentiation is abnormal. In contrast to AMKL, TL undergoes spontaneous remission within the first 3 months. In approximately one fifth of TL cases, however, AMKL develops later in life. Acute megakaryoblastic leukemia (AMKL) is a form of leukemia where a majority of the blasts are megakaryoblastic. This category of AML is associated with $30 \%$ or more blasts in the marrow. Blast are identified as being of megakaryocyte lineage by Expression of megakaryocyte specific antigens and platelet peroxidase reaction on electron microscopy. It is associated with GATA1, and risks are increased in individuals with Down syndrome[9] . However, not all cases are associated with Down syndrome and other genes can also be associated with AMKL. Another related gene is MKL1, which is also known as "MAL". This gene is a cofactor of serum response factor. In the first three years of life megakaryoblastic leukemia is the most common type of leukemia in patients with Downs syndrome. AML-M7 itself is a rare neoplasm. Risk of AML-M7 is 150 times more in Down's children than general population[10]. No obvious relationship was found between haemoglobin E beta thalassaemia and AML M7 till date and so we report our case as an incidental findings with this trio (Down's syndrome, HbE beta thalassaemia and AML M7) for future research .

\section{HEADINGS}

1.Case History :A 1 year 8 months old male child attended our OPD with a complain of malaise, fever, progressive pallor for 2 months and swelling of abdomen and both legs with progressively increasing shortness of breath for last 15 days. No history of any bleeding manifestation, seizure, jaundice, any contact with tuberculosis, previous history of blood transfusion or any other sibling affection was present. Child however had a history of developmental delay.

\section{Examination :}

2.1 General Survey :Patient had tachypnea, tachycardia, fever, severe pallor, bilateral pitting pedal oedema and bony tenderness. Cyanosis, icterus and significant lymphadenopathy was absent. Head to foot examination revealed brachycephaly, depressed nasal bridge, flat occiput, epicanthic folds, speckeled iris, Bilateral simian crease. 
2.2 Systemic Examination :Abdomen distended, soft, everted umbilicus with full flanks. Liver-4 $\mathrm{cm}$ palpable below right costal margin along mid clavicular line with span of $9 \mathrm{~cm}$ which was soft, smooth, and tender with rounded margin. Firm spleen was palpable $4 \mathrm{~cm}$ along its axis. Abdomen showed presence of ascitis.CVS examination revealed hyperdynamic precordium with cardiomegally, presence of ejection systolic murmur (haemic murmur) in aortic area with S3 gallop. Chest examination showed bilateral basal crepitations.

3.Management : Immediate supportive treatment was started along with few basic investigations. Complete heamogram showed Haemoglobin 3.4gm/dl, Total leucocyte count 33,000/cmm, DLC showed Neutrophils $20 \%$, Lymphocytes $21 \%$,Monocytes $1 \%$, Blast cells $58 \%$, Platelet count $10,000 / \mathrm{cmm}$, Erythrocyte count $1.30 \mathrm{million} / \mathrm{cmm}$, PCV 11\%, Reticulocyte count $2.2 \%$, MCV $84.5 \mathrm{fl}, \mathrm{MCH} 26.1 \mathrm{pg}$, MCHC 30.9\%, RDW-CV $24.4 \%$. Peripheral blood smear shows RBC, predominantly normocytic, normochromic, anisocytosis and few tear drop cells. After patient stabilization with blood products and supportive treatment, bone marrow biopsy was done which shows diffuse marrow infiltration by immature cells having irregular nuclei, dispersed chromatin, indistinct nucleoli and scanty irregular cytoplasm. Normal haematopoiesis is markedly suppressed. Bone marrow smear shows $70 \%$ blast cells (fig 1). Reticulin stain showed intersecting coarse reticulin fibres. Immunophenotyping of haematopoietic neoplasms was done from peripheral blood sample. Applying appropriate gate on approximate 50,000 leucocytes in the CD 45/SSc plot an abnormal expansion of a population of progenitor cells with moderate $\mathrm{CD} 45$ and side scatter is detected showing expression of cytoplasmic CD 41a and CD 61 with absent Cmpo, CD36, CD9, CD71, CD13, CD33, CD117, with minimal CD38 and CD123. CD4, CD7, CD56, CD34 expression is absent in these cells. These cells constitute $32 \%$ of all leucocytes. Features are consistent with acute megakaryoblastic leukaemia. Chromosome analysis was done from peripheral blood, using 72 HRS stimulated culture-GTG banding showed karyotype 47 xy, +21(fig 2). Post transfusion HPLC of the patient showed ,Hb F 10.8\%, P2 3.4\%, P3 4\%, Hb Ao58.2\%, Hb A2 +E 22.7\% with absent $\mathrm{Hb} \mathrm{S}$ and $\mathrm{Hb} \mathrm{D}$ (fig 3). HPLC of both the parents were done- Father was beta thalassaemia trait while mother was Haemoglobin E trait. Patient was treated with a chemo regime of cytosine and arabinoside. Patient responded well and now is under follow up.

III. FIGURES AND TABLES

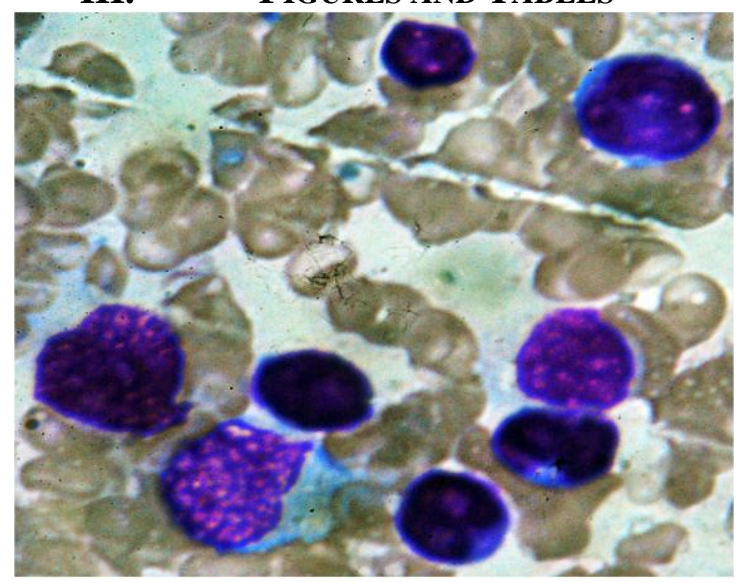

Figure 1: Bone marrow smear showing megakaryoblast

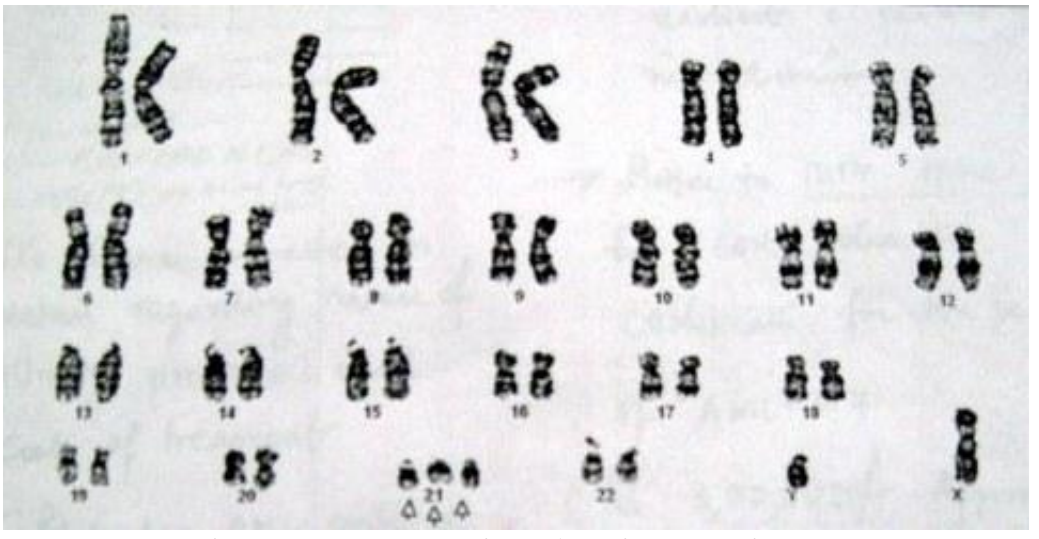

Figure 2: Karyotyping showing 21 trisomy 


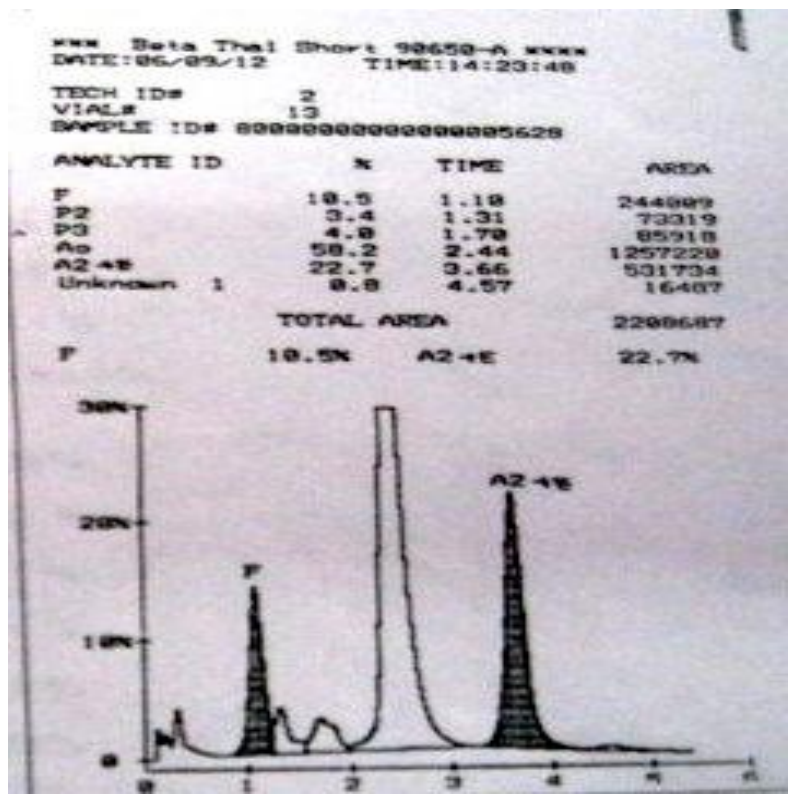

Figure 3.HPLC of the patient

\section{CONCLUSION}

Here we found a 1 year 8 month old child of Down's syndrome having Acute megakaryoblastic leukemia and Haemoglobin E beta thalassaemia coexisting together. Though, association of acute myeloblastic leukemia is quite common in Down's baby and has quite high incidence but association of Down's syndrome with Haemoglobin E beta thalassaemia has not been found. This finding of appearance of triad in a single child viz. Down's syndrome, acute megakaryoblastic leukemia and Haemoglobin E beta thalassaemia may be incidental but gives the message that this type of association may exist and should be sought for and must be reported for future research.

\section{REFERENCES}

[1] Fong CT, Brodeur GM. Down's syndrome and leukemia: epidemiology, genetics, cytogenetics and mechanisms of leukemogenesis. Cancer Genet Cytogenet. 1987;28:55-76

[2] Robison LL. Down syndrome and leukemia. Leukemia. 1992;6(suppl 1):5-7.

[3] Robison LL, Nesbit ME Jr, Sather HN, et al. Down syndrome and acute leukemia in children: a 10-year retrospective survey from Childrens Cancer Study Group. J Pediatr. 1984;105:235-242.

[4] Bennett JM, Catovsky D, Daniel MT, et al. Criteria for the diagnosis of acute leukemia of megakaryocyte lineage (M7): a report of the

[5] French-American-British Cooperative Group. Ann Intern Med. 1985;103:460-462 Zipursky A, Poon A, Doyle J. Leukemia in Down syndrome: a review. Pediatr Hematol Oncol 1992;9:139-149

[6] Zipursky A. Transient leukemia - a benign form of leukemia in newborn infants with trisomy 21. Br J Haematol. 2003;120:930-938.

[7] Gamis AS, Hilden JM. Transient myeloprolifera-tive disorder, a disorder with too few data and many unanswered questions: does it contain an important piece of the puzzle to understanding hematopoiesis and acute myelogenous leukemia? J Pediatr Hematol Oncol. 2002;24:2-5.

[8] Taub JW, Ravindranath Y. Down syndrome and the transient myeloproliferative disorder: why is it transient? J Pediatr Hematol Oncol. 2002;24:6-8.

[9] Hitzler JK, Cheung J, Li Y, Scherer SW, Zipursky A (2003). "GATA1 mutations in transient leukemia and acute megakaryoblastic leukemia of Down syndrome". Blood 101 (11): 4301-4

[10] Heim, S., Mitelman, F., 1986. Numerical chromosome aberrations in human neoplasia. Cancer Genetics and Cytogenetics, 22(2):99108 